\title{
LA GÉNESIS DE LA «PRUEBA» RACIONAL DE LA EXISTENCIA DE DIOS EN XAVIER ZUBIRI A LA LUZ DE SUS ÚLTIMOS ESCRITOS ${ }^{1}$
}

\author{
ESTEBAN VARGAS \\ Pontificia Universidad Católica de Valparaíso
}

\begin{abstract}
RESUMEN: En este trabajo intentamos mostrar cómo Zubiri fue entendiendo y precisando su «prueba» o argumento de la existencia de Dios desde sus últimas obras. Estudiamos su prueba a través de la "vía de la religación" desde cuatro textos o cursos: El problema teologal del hombre: Dios, religión, cristianismo (1971), El despliegue del problema de Dios (1971-1973, publicado como segunda parte en El hombre y Dios), El problema teologal del hombre: El hombre y Dios (1973) e Inteligencia sentiente (1980-1983).

PALABRAS CLAVES: Dios; Zubiri; religación; prueba; razón; razonamiento.
\end{abstract}

\section{Genesis of the rational "proof» for the existence of God in Xavier Zubiri in light of his last writings}

ABSTRACT: This paper attempts to show how Zubiri was understanding and explaining his "proof» or argument for the existence of God from his last works. We study his proof through the «way of religation» from four texts or seminars: The theological problem of man: God, religion, Christianity (1971), The unfolding of the problem of God (1971-1973, published as a second part in Man and God), The theological problem of man: man and God (1973) and Sentient Intelligence (1980-1983).

KEY WORDS: God; Zubiri; religation; proof; reason; reasoning.

\section{INTRODUCCIÓN}

En 1984 se publicó póstumamente el libro El hombre y Dios (HD) del filósofo español Xavier Zubiri (1898-1983). Contiene, entre otros temas, en su Segunda Parte, una brillante prueba o argumento sobre la existencia de Dios que Zubiri obtiene por lo que él llama la «vía de la religación o de la realidad». Lamentablemente, el libro no pudo terminarse por la muerte del autor. Zubiri logró concluir, en su último año de vida, sólo la Primera Parte y el inicio de la Segunda. La Segunda Parte publicada en la primera edición de 1984 procede de textos anteriores a esa Primera Parte y, por tanto, requería de una última redacción donde Zubiri seguramente actualizaría esa "prueba» a la luz de todo lo pensado en su ya clásica última obra Inteligencia sentiente (IS) en tres tomos (1980-1983), tal como lo había alcanzado a hacer con la Primera Parte. ¿Cómo poder entender esta importante prueba y actualizarla en función de sus últimas obras? La investigación que realicé como nuevo editor de HD, libro ya publicado ${ }^{2}$, ha permitido conocer nuevos textos de esa Segunda Parte, los que ofrecen nuevas pistas para tratar de responder tal pregunta.

1 Este artículo fue posible gracias a la Fundación Xavier Zubiri de Madrid, que permitió el acceso a archivos inéditos.

2 Zubiri, X. (2012). El hombre y Dios. Nueva edición. Madrid: Alianza. 
El propósito de este trabajo es ofrecer, entonces, la génesis o evolución de esta prueba en la etapa final de su producción filosófica que va de 1971 en adelante $^{3}$. En esta etapa disponemos de cuatro textos desde donde podemos intentar meditar tal prueba en su interno despliegue. En primer lugar, la Primera Parte del curso El problema teologal del hombre: Dios, religión, cristianismo (PTH), dictado en 1971, y que hace unos años hemos publicado en forma completa junto a Antonio González $z^{4}$ Este curso nos presenta el primer esbozo de un ambicioso plan de Zubiri en tres partes en torno al problema de Dios. La Primera Parte de ese curso contiene la «prueba» o argumento de Dios. En segundo lugar, hay que considerar un extenso escrito mecanografiado (con varias correcciones manuscritas de Zubiri) que pertenece a la Segunda Parte del libro HD. Lo llamamos en la nueva edición de HD «El despliegue del problema de Dios» (DPD). En tercer lugar, poseemos el curso El problema teologal del hombre: El hombre y Dios (PTHHD) de 1973, que desarrolla y amplía la Primera Parte de PTH. La Segunda Parte de este curso de 1973 desarrolla la «prueba» de Zubiri, y existen varias razones, como veremos más abajo, para creer que contiene la última versión escrita de ella. Este curso fue publicado íntegramente en la nueva edición de HD. Finalmente, hay que considerar su gran libro Inteligencia sentiente, publicado entre 1980-1983, que, aunque no explicite tal prueba, sí da algunas pistas de cómo podría ser repensada.

Antes de desarrollar la génesis de la «prueba» de Dios en Zubiri, convendría tener en cuenta algunos aspectos que orientan la marcha de este escrito.

En primer lugar, la «prueba» presupone como «punto de partida» un análisis descriptivo de la religación, en definitiva, de cómo el hombre hace su vida. Este análisis constituye lo que Zubiri llama muchas veces «el problema de Dios», expresión que puede llevar al error, si se entiende por «Dios», un ser supremo «concreto», por ejemplo, el Dios de una religión determinada ${ }^{5}$. El análisis de la religación nos muestra algo a lo cual «ya» tenemos acceso. En general, aunque con algunos matices, este "punto de partida» es descrito en forma semejante «en sus últimas obras». No es tema de este artículo desarrollar este punto. Sin embargo, para orientación del lector, puede decirse a grandes rasgos que el "punto de partida» consiste básicamente en lo siguiente:

1. Al hombre, por su inteligencia sentiente, se le presentan diversas cosas reales con las cuales hace su vida. Estas cosas reales poseen un momento transcendental por el cual son algo «más» que el contenido de lo que se presenta.

3 Hasta el curso del año 1968 llamado «El hombre y el problema de Dios» (recientemente publicado en Sobre la religión. Madrid: Alianza, 2017), Zubiri utiliza como prueba lo que podría llamarse la vía cosmológica. No es tema de este artículo estudiar esta vía cosmológica, sino la vía de la religación.

4 Zubiri, X. (2015). El problema teologal del hombre: Dios, religión, cristianismo. Madrid: Alianza.

5 Para más detalle sobre lo que significa la expresión «el problema de Dios» en Zubiri puede verse Vargas, E. (2015). «Filosofía y el problema de Dios» en Pensamiento, vol. 71, núm. 266, pp. 491-504. 
«Estamos con las cosas, pero donde estamos con ellas es en la realidad» ${ }^{6}$. La realidad como formalidad es «más» que sus contenidos, aunque no es un «más» sin ellos. Este momento de «más» domina, tiene «poder» sobre sus contenidos. Este poder de lo real, domina, se impone, lanzándonos a realizarnos.

2. El hombre para poder realizarse se apoya «en» la realidad en tanto realidad como fundamento. La realidad como fundamento no es algo que meramente esté ahí, no es una realidad-objeto, sino que nos impele a realizarnos. «Esta peculiar ligadura es justo religación $»^{7}$. El hombre se encuentra religado al poder de lo real. Esta realidad-fundamento (o fundamentalidad como la llama con más rigor Zubiri) a la que el hombre está religado posee un carácter último, posibilitante e impelente. La realidad es "última», en tanto que el hombre hace su vida «en» ella. Es «posibilitante» en tanto hace su vida «desde» ella. Finalmente, es «impelente», en tanto hace su vida "por» ella.

3. Esta religación al poder de lo real, a la realidad-fundamento, es «problemática» o ambivalente. Ello porque son las cosas reales las que nos impelen a tener que realizarnos, pero ninguna de ellas es «la» realidad.

En esto consiste básicamente el punto de partida.

En segundo lugar, hay que aclarar que la «prueba» no es un camino que, como "punto de llegada», nos lleva a un Dios extramundano que «no» se encuentra en la aprehensión, sino que más bien es una vía que nos «actualiza» el «contenido fundamental» de una «realidad» en que «ya» estamos ${ }^{8}$. Así, nos dirá Zubiri que «la prueba no lo es tanto de que hay Dios, sino de que algo de lo que hay realmente es Dios» ${ }^{9}$. Por ello, la «prueba» no pretende demostrar la existencia de una realidad más, distinta de todas las ya aprehendidas, sino sumergirnos en la formalidad de realidad ya dada.

En tercer lugar, esta actualización del «contenido» de la realidad-fundamento (el punto de llegada de la prueba) no se obtiene como producto de una mera descripción. En esto se diferencia precisamente del «punto de partida». ¿Cómo se obtiene, entonces, esta actualización fundamental? Para Zubiri, ya como vimos en la nota, desde Naturaleza, Historia, Dios, esta actualización se obtiene a través de un «razonamiento», de una intelección de-mostrativa. Una

6 ZuBIRI, X. (2012). El hombre y Dios, op. cit., p. 88.

7 Ibíd., p. 98.

8 La idea de que la prueba de Dios presupone una cierta «actualidad» de Él está ya presente, en cierto modo, desde Naturaleza, Historia, Dios, cuya primera edición es de 1944. Así Zubiri nos dice: "Cuando tratamos de elevarlo a concepto [Dios] y de darle justificación ontológica, entonces, y sólo entonces —es decir, supuesta esta visión, supuesta la religación-, es cuando nos vemos forzados a intentar una demostración discursiva de la existencia y de los atributos entitativos y operativos de Dios. Tal demostración no sería jamás el descubrimiento "primario» de Dios [...] Nuestro análisis no sólo no ha hecho inútil la marcha del entendimiento hacia Dios, sino que la ha exigido necesariamente. Recíprocamente, el hecho de que el entendimiento humano posea la nuda facultad de demostrar la existencia de Dios jamás significaría que sea el discurso la primera vía de llegar intelectualmente a ella" (Zubiri, X. (1987). Naturaleza, Historia, Dios. Madrid: Alianza, p. 444).

9 ZuBiRI, X. (2012). El hombre y Dios, op. cit., p. 245. 
intelección «mostrativa» muestra apuntando o describiendo un «principio» del conocimiento, en este caso, la religación al poder de lo real como realidadfundamento. Una intelección «de-mostrativa» pretende, por conclusión de un razonamiento, mostrar una realidad, en este caso, el «contenido» de la realidadfundamento, de Dios, «desde» sus premisas o principios, en este caso, desde la religación. ¿Qué papel juega el razonamiento en la prueba de Dios de Zubiri? Anticipando ideas, hay que señalar que el razonamiento podría jugar, al menos, dos grandes papeles en la prueba de Dios.

a) Apoyados en lo aprehendido (religación), la conclusión del razonamiento verifica "directamente» el contenido fundamental de algo que no podemos aprehender o percibir. Ésta «parece» ser la tesis de Zubiri de los primeros cursos de los años setenta.

b) Apoyados en lo aprehendido (religación), la conclusión del razonamiento nos sirve como «medio» para obtener un «esbozo» o posibilidad de lo que «sería» Dios, todo lo cual debe ser verificado en la experiencia. Es la tesis, por ejemplo, de Gracia (2007) y Sáez (1995), entre otros, la cual es obtenida a la luz de Inteligencia sentiente, pero sin respaldo explícito en lo publicado en la primera versión de HD.

Cabría meditar si la prueba de Dios, en el último Zubiri, no es, en rigor, un razonamiento, sino más bien un proceso elaborativo de «libre creación».

Veamos ahora la génesis o marcha de la prueba de la existencia de Dios a través de la vía de la religación en Zubiri en sus textos y cursos a partir de los años setentas. Es lo que intentaremos desarrollar ahora.

\section{1.- El PROBLEMA TEOLOGAL DEL HOMBRE: Dios, RELIGIÓN, CRISTIANISMo (1971)}

Una primera versión de la "prueba» de Dios, según la «vía de la religación», la encontramos en la Primera Parte de este curso de 1971, recientemente publicado. En la sexta lección (con algunos intercalados mecanografiados que corrigen lo expuesto en sus lecciones), Zubiri señala que la prueba se produce por un razonamiento que posee dos partes.

La primera parte de ese razonamiento (que presupone, como punto de partida, el análisis de la religación y, por tanto, la distinción entre cosas reales y «la» realidad) se divide, a su vez, en dos proposiciones que pueden sintetizarse como sigue:

1) Todo poder se funda en una realidad.

2) El poder no puede fundarse en «cada una» de las cosas reales ni en su mero conjunto.

Por tanto, la conclusión es, entonces, que el poder se funda en «la» realidad entendida como realidad absolutamente absoluta. Zubiri lo señala así:

«La primera parte del razonamiento se reduce, pues, a estas dos proposiciones con su correspondiente conclusión: el poder se funda en la realidad, y la 
realidad en que se funda ese poder no son exclusivamente las cosas reales con que yo tropiezo. Luego, tiene que ser necesariamente una realidad absolutamente absoluta. Esta realidad, por tanto, existe» ${ }^{10}$.

La segunda parte del razonamiento, que es más bien un análisis, intenta mostrar que esa realidad absolutamente absoluta no es otra cosa que lo que todos entendemos por Dios. Así, Zubiri nos dice: «Segunda parte del razonamiento. Y esta realidad es idénticamente Dios. No por una inferencia, ésta es la segunda parte del razonamiento, sino pura y simplemente por un análisis de la vía que hemos seguido» ${ }^{11}$.

Este análisis nos muestra que lo que se entiende «nominalmente» por Dios en la historia de las religiones (un Dios al que se le reza, etc.) corresponde a lo que «realmente» se ha encontrado en la primera parte del razonamiento. Todo ello lo resume Zubiri así:

«Basta abrir un libro de historia de las religiones; ¿qué se entiende por Dioses? Efectivamente, eso. No solamente unos seres supremos, sino unos seres supremos a quienes el hombre puede dirigirse de una cierta manera, de quienes puede recibir ayuda, en quienes puede uno ayudarse, con éxito o sin él, pero, en fin, esto es lo que entendemos por Dios. Y esto que hasta ahora no era más que una definición nominal de Dios, se manifiesta, gracias a la primera parte del razonamiento, que es idénticamente una definición real. Real y efectivamente, la realidad absolutamente absoluta es el fundamento último de la ultimidad, de la posibilidad y de la impelencia. Esto es formalmente Dios en tanto que Dios ${ }^{12}$.

La primera parte del argumento, el razonamiento en rigor, parece intentar demostrar, a partir de la experiencia de la religación, la existencia de una realidad absolutamente absoluta. Es la definición real de esta realidad. En la segunda parte del argumento se identifica esta realidad con lo que entendemos nominalmente por Dios. Por ello, en este argumento se pretende haber logrado «de-mostrar» realmente la existencia de la realidad absolutamente absoluta (Dios). Al parecer, se ha logrado verificar «directamente» el «contenido» de Dios en tanto que Dios (ser realidad absolutamente absoluta) por razonamiento. Pero Zubiri, a nuestro entender, siguió precisando el argumento.

\section{2.- El Despliegue del PRoblema de Dios (1971-1973)}

La segunda versión del argumento la encontramos en un escrito mecanografiado que llamamos «El despliegue del problema de Dios» (DPD) y que fue publicado como Segunda Parte de HD, tanto en la primera edición como en la última que yo he podido editar. Lo citaremos, entonces, desde la

10 ZubiRI, X. (2015). El problema teologal del hombre: Dios, religión, cristianismo. Madrid: Alianza, pp. 111-112.

11 Ibíd., p. 112.

$12 \quad$ Ibíd., pp. 112-113. 
nueva edición de HD. Según Ellacuría, el primer editor de HD, este texto se escribió con posterioridad al curso de 1973 llamado El problema teologal del hombre: El hombre y Dios (PTHHD) ${ }^{13}$, curso que estudiaremos más abajo. Hay muchas pistas que muestran que esto no es correcto, al menos, en la parte donde se desarrolla esta "prueba». Por esto, su fecha de creación debe situarse, tal vez, entre 1971 y 1973. Veremos algunas de estas razones más abajo. Por ahora, conviene aclarar cómo se entiende y desarrolla aquí este argumento.

En primer lugar, el argumento o prueba sigue calificándose como un razonamiento o demostración, proceso que se califica como el modo «más estricto y constringente de intelección» ${ }^{14}$. Sin embargo, esta prueba no es entendida como un mero momento demostrativo, sino que está colocada más explícitamente ahora dentro de la marcha de la vida personal, donde hay que incluir también el momento volitivo u opcional. La prueba, más que un mero razonamiento, es ahora más claramente explicitada como un "proceso intelectivo» donde la opción por el fundamento (que Zubiri llama voluntad de fundamentalidad) juega un papel crucial $^{15}$. Junto a la intelección sentiente de fundamentalidad, hay una voluntad tendente de fundamentalidad. Ello ocurre porque la fundamentalidad, por su propio carácter, impele a ser inteligida y optada inexorablemente. Expliquemos un poco este papel de la volición en la "prueba». La voluntad de fundamentalidad es, para Zubiri, el "principio radical de una actitud [teísmo, ateísmo, etc.] que desde sí misma se despliega en proceso intelectivo y opción» ${ }^{16}$. Esta volición radical es principio de todo el "proceso sentiente», volición que, como vimos en la cita anterior, posee dos aspectos.

Así, por una parte, esta volición es principio de «intelección» ulterior, de lo que después, en IS, Zubiri llamará el logos y la razón. Así nos dice: «La voluntad de fundamentalidad no es meramente un acto consecutivo a la intelección de la realidad-fundamento, sino que es una voluntad que pone en marcha el proceso intelectivo ${ }^{17}$. Ciertamente, esta voluntad no determina por sí sola la verdad de una prueba de Dios. En palabras de Zubiri:

«La prueba de la existencia de Dios, es decir, su intelección demostrativa [por razonamiento], aun puesta en marcha por la voluntad de verdad, tiene un alcance y un valor que no penden de esta voluntad sino sola y exclusivamente de la inteligencia misma. La discusión acerca de la validez de las

13 Cf. Zubiri, X. (1984). El hombre y Dios. Primera Edición. Madrid: Alianza, p. v.

14 ZubiRI, X. (2012). El hombre y Dios, op. cit., p. 282.

15 Esto parece explicar, por ejemplo, una nota que Zubiri puso al margen de este texto y que fue incorporada por Ellacuría directamente al texto del libro de la Segunda Parte de HD, en la primera edición. En ella Zubiri nos dice: «Esta "prueba" no es como una demostración matemática. Tiene siempre la resonancia de la marcha de la vida personal. Y, por tanto, sólo resulta completa en el desarrollo entero del libro. El libro es lo que da a esta prueba el carácter probatorio de una experienciación física» (ZubiRI, X. (2012). El hombre y Dios, op. cit., p. 168).

16 Zubiri, X. (2012). El hombre y Dios, op. cit., p. 299.

17 Ibíd., p. 271. 
pruebas es asunto de intelección y nada más [...] Lo que no está dicho es que proceso intelectivo sea idéntico a demostración ${ }^{18}$.

La voluntad de fundamentalidad, para Zubiri, está fundada sobre el hecho primordial de la religación (aquí no hay opción), pero su carácter "problemático» nos abre a tener que tomar una "opción concreta», por ejemplo, desde qué aspectos vamos a intentar «describir» la realidad-fundamento (logos), de qué modo intentaremos «conocerla» (razón), etc. La volición juega un papel importante en el proceso intelectivo y, por tanto, en la «prueba» de Dios.

Por otra parte, esta volición radical, apoyándose en lo actualizado por todo el proceso intelectivo, es también principio de «apropiación» opcional de un modo de «entrega» a la realidad-fundamento. En este escrito no intentaremos explicar «toda» la marcha personal del hombre a Dios, sino sólo el aspecto «intelectivo» de esa marcha.

En segundo lugar, veamos ahora cómo Zubiri desarrolla su argumento demostrativo. Ante todo, hay que señalar que Zubiri no expresa con claridad los límites del punto de partida y de llegada del argumento. Zubiri enuncia el proceso intelectivo en cuatro pasos, donde el último paso parece ser el propiamente demostrativo. Si nos concentramos en ese último paso, vemos que allí ya no se muestran los límites exactos de las dos partes en que se exponía el argumento, según el curso de 1971. Al parecer, Zubiri intenta mostrar el proceso intelectivo como algo más cohesionado, sin los límites forzados que puede dar un análisis racional dividido en premisas.

¿Cómo se explica básicamente este argumento demostrativo? Partiendo del análisis de la religación, Zubiri señala, al igual que en el curso de 1971, que el carácter enigmático de lo real, por su poder, nos remite al fundamento en que se apoya ese poder. Ello porque ese poder, ese ser "más» (lo que tiene de realidad cada cosa real) no le viene de sí mismo por cuanto el momento de realidad es algo que excede del «dominio» de cada cosa real. Ese poder entonces, para Zubiri, «debería fundarse» en una realidad distinta de las que tenemos ahí presentes por cuanto todo "poder» se funda en algo "de suyo». Así, nos dice Zubiri que «el poder de lo real, en efecto, como todo poder, se funda en las propiedades (digámoslo así) que posee la cosa que tiene tal poder; si no las poseyera, no tendría ese poder. Y aquí está el nudo de la cuestión ${ }^{19}$. Las cosas reales, entonces, se fundan, no en otras cosas reales presentes, dado que el momento de realidad excede de todas ellas, sino en una realidad que sea infundada, en una realidad que Zubiri llama «absolutamente absoluta». Esa realidad-fundamento o fundamentalidad de la que hemos partido es entendida por razonamiento como «realidad absolutamente absoluta». Es justo lo que Zubiri sostiene que todos entendemos por Dios (por ejemplo, en las religiones). Es algo «más» que cada cosa real, pero es un «más» en ellas mismas. Zubiri resume todo esto así: «el poder de lo real se funda esencialmente en la

$18 \quad$ Ibíd., pp. 281-282.

19 Ibíd., pp. 164-165. 
índole misma de la realidad. Luego, este poder está fundado en una realidad absolutamente absoluta, distinta de las cosas reales, pero en las cuales, por serlo, está formalmente constituyéndolas como reales. Esta realidad es, pues, Dios $»^{20}$. Es interesante destacar que donde en la cita dice «luego», Zubiri la ha subrayado a mano, y cuando ha presentado este mismo argumento, unas páginas antes, Zubiri ha anotado al margen: "Ojo con el "luego" $»^{21}$. Esta anotación se entenderá mejor cuando expongamos este argumento a la luz del curso de $1973^{22}$.

\section{3.- El problema teologal del hombre: El hombre y Dios (1973)}

La tercera y última versión del argumento que conservamos la encontramos en la Segunda Parte de este curso de 1973. De él conservamos la versión mecanografiada de las lecciones orales, corregida por Zubiri, más los «Apuntes o Fichas» que le servían de guía para la exposición del curso. Este curso ha sido publicado íntegramente en la nueva edición de HD desde donde lo citamos. Ahora bien, estas lecciones traen como novedad, para el tema de este escrito, dos ideas importantes.

a) Por una parte, Zubiri ya no afirma tan tajantemente como antes que su prueba o argumento de Dios sea una «demostración». Así, nos dice: «No insisto en que [esta prueba] sea una demostración. Si es o no demostración, diría un griego, $\pi \mathrm{o} \lambda \lambda \alpha \chi \tilde{\omega} \varsigma \lambda \varepsilon ́ \gamma \varepsilon \tau \alpha$, se dice de muchas maneras qué puede ser una demostración. Hablemos de intelección procesual en el sentido más amplio del vocablo» ${ }^{23}$.

Esta «intelección procesual», si bien ya no es claramente identificable con una demostración, sigue conservando su carácter de algo que resulta de un esfuerzo intelectual (que Zubiri llama interpretación) que nos lleva al «contenido concreto» de Dios, sin ser tal esfuerzo un hecho como la religación. Así, Zubiri nos dice:

«Ahora bien, si la religación es un hecho, el arribar a Dios, por consiguiente, como acabamos de recordar, no lo es. Necesito un esfuerzo intelectivo, una interpretación, todo lo fundada que se quiera, pero, en fin, no es un hecho.

$20 \quad$ Ibíd., p. 167.

21 Ibíd., p. 165.

22 A mi juicio, después de demostrar la existencia de Dios, Zubiri, en un segundo paso racional, deduce los caracteres «concretos» de Dios. Para Zubiri, Dios, por ser realidad absolutamente absoluta, tiene que ser una realidad personal (suya), inteligente y volente. Por ello, nos dice: «En definitiva, Dios es realidad absolutamente personal, en actividad de vida absoluta, auto-actual en inteligencia y voluntad. He aquí la absoluta concreción de la realidad absolutamente absoluta» (ibíd., p. 188).

23 Ibíd., p. 457. 
Dios, por consiguiente, no está directa y formalmente accedido en este proceso intelectivo ${ }^{24}$.

Es importante destacar que cuando Zubiri debía explicar en el curso de 1973 el argumento o prueba de Dios, en sus «Fichas» tenía anotado el número de unas páginas que corresponden exactamente a las del texto «El despliegue del problema de Dios» (DPD) ya mencionado, donde también se expone esta prueba. Además, llevaba anotada en sus «Fichas» la parte esencial del argumento que coincide casi literalmente con lo expresado en tal texto. Esto es una pista de que el curso PTHHD de 1973 es "posterior» a DPD, al menos en este punto. También es importante destacar que, en sus «Fichas», la «conclusión» de la prueba se encuentra tachada, conclusión que corresponde casi literalmente a lo expuesto en DPD y que se iniciaba con un «luego», como veíamos antes. Zubiri, cuando planificó el curso de 1973, pensó probablemente en decir lo expuesto en DPD, pero a última hora se arrepintió. Ahora se podría intentar interpretar esta tachadura. Zubiri parece querer quitar ese carácter de razonamiento, de premisas y conclusión definidas que tenía el argumento de Dios, lo cual quedaría plasmado al decir «luego,...». Además, esa tachadura (más lo que diremos a continuación) nos sirve nuevamente para mostrar que el curso de 1973 es «posterior» al texto DPD.

b) Por otra parte, hay que señalar que Zubiri, en sus «Fichas», reemplaza el texto tachado recién mencionado con una nueva hoja que lleva la página 25 «bis», lo que demuestra que es un agregado de última hora. Esta hoja es la que Zubiri termina finalmente pronunciando en el curso de 1973. El texto del curso dice así:

«Toda realidad tiene poder, pero ninguna cosa concreta es la que da el poder de la realidad a estas cosas concretas, sino que es "la" realidad lo que da poder a cada cosa concreta. Ese poder de la realidad es real, y necesita fundamentarse realmente en algo que no puede ser otra realidad concreta más, porque, si no, estaríamos en el mismo caso que antes. Y como esta realidad debe fundamentar absolutamente mi realidad relativamente absoluta, esta realidad no solamente tiene que ser real, sino que es una realidad absoluta, es decir, es un fundamento absoluto de realidad, es una realidad absolutamente absoluta. Justo ésta es la esencia de Dios» ${ }^{25}$.

Este texto, además de quitar el «luego», explicita que el poder de lo real no puede fundarse en «cada» cosa real, dado el carácter de enigma o ambivalencia de la realidad. Toda realidad es «más» que cada cosa real, un «más» que se da en ella misma. Hasta aquí ha dicho esencialmente lo que ha afirmado en los textos anteriores. Lo que Zubiri ahora explicita un poco más es que si el poder de lo real estuviera fundado en una cosa real concreta, esa misma cosa real reclamaría, a su vez, su fundamento en otra cosa real y así sucesivamente. Como la fundamentación no puede ir a dar al infinito, debe fundarse en una realidad absolutamente absoluta (Dios). Este argumento se parece a los argumentos

24 Ibíd., p. 481.

25 Ibíd., p. 464. 
clásicos de la existencia de Dios, específicamente a los de santo Tomás ${ }^{26}$. Zubiri, de hecho, parece insinuarlo en el siguiente pasaje del curso:

«Esta realidad absolutamente absoluta es justamente Dios. Porque no se conseguiría nada, repitan ustedes aquí el argumento clásico, si ese fundamento fuese a su vez una cosa real; más bien de esta cosa real diríamos lo que acabamos de decir, que necesita un fundamento, porque sería ambivalente ${ }^{27}$.

Sin embargo, Zubiri muestra algunas diferencias con la vía de santo Tomás. Zubiri, ante todo, no llega a Dios por la vía de la causalidad eficiente o de otro tipo, sino por la vía de la realidad, específicamente, por la vía de la dominancia o poder de lo real. Dios no es formalmente, en rigor, la causa primera eficiente, sino el fundamento último, posibilitante e impelente de lo real. Además, el argumento o prueba de la existencia de Dios, en este curso, no parece ser, en rigor, una demostración como los argumentos de santo Tomás. Pero eso no es algo tan claro en este curso de 1973. Esto es algo que debe ser aclarado.

Posterior al curso de 1973, Zubiri meditó por largos años sobre los procesos intelectivos, lo que quedó expresado finalmente en su libro IS. Sin duda, a la luz de lo obtenido en esta obra, Zubiri habría podido plantear mejor lo expresado en el curso de 1973. Esto pudo hacerlo en la Primera Parte de ese curso, pero por su muerte no alcanzó a hacerlo en la Segunda Parte donde se debía volver a redactar la «prueba» de Dios. Por ello, sólo cabe aquí la especulación de cómo podría haber sido desarrollada esta parte fundamental de su obra sobre Dios.

\section{IV- InTEligencia SENTIENTE (1980-1983)}

En un curso llamado «La inteligencia humana» (1976), Zubiri comenzó, en su última etapa filosófica, un análisis de la intelección que prosiguió en sus seminarios privados y que desembocó finalmente en una extensa obra de tres tomos titulada Inteligencia sentiente (IS). Este tema concentra todo el interés de Zubiri desde el año 1976 en adelante de tal modo, que no dicta ningún curso más para el público. En esta obra, Zubiri, como decíamos, no explicita su argumento de Dios. En este sentido, IS no puede darnos ninguna información

26 Compárese, por ejemplo, el argumento de Zubiri con la segunda vía de santo Tomás: «La segunda es la que se deduce de la causa eficiente. Pues nos encontramos que en el mundo sensible hay un orden de causas eficientes. Sin embargo, no encontramos, ni es posible, que algo sea causa eficiente de sí mismo, pues sería anterior a sí mismo, cosa imposible. En las causas eficientes no es posible proceder indefinidamente porque en todas las causas eficientes hay orden: la primera es causa de la intermedia; y ésta, sea una o múltiple, lo es de la última. Puesto que, si se quita la causa, desaparece el efecto, si en el orden de las causas eficientes no existiera la primera, no se daría tampoco ni la última ni la intermedia. Si en las causas eficientes llevásemos hasta el infinito este proceder, no existiría la primera causa eficiente; en consecuencia no habría efecto último ni causa intermedia; y esto es absolutamente falso. Por lo tanto, es necesario admitir una causa eficiente primera. Todos la llaman Dios»: SANTO Tomás, (2007). Suma de Teología. Madrid: BAC, I q 2, a 3.

27 Zubiri, X. (2012). El hombre y Dios, op. cit., p. 481. 
concluyente de cómo, en definitiva, Zubiri habría repensado su «prueba» de Dios. Sin embargo, podríamos decir que buena parte de IS está escrita con vistas a HD. De hecho, apenas terminada esa obra, Zubiri se pone a re-escribir inmediatamente HD alcanzando a concluir la Primera Parte y el comienzo de la Segunda (publicada sólo en la nueva edición) que, dicho sea de paso, se parece bastante más al comienzo de la Segunda Parte del curso de 1973 que al texto DPD. Hay muchos análisis que se hacen en IS y que serían muy importantes para repensar lo dicho en HD como, por ejemplo, la idea de realidad y poder, los momentos de la inteligencia sentiente, el análisis de la realidad-fundamento, etc. No es nuestro interés intentar reactualizar completamente HD a la luz de IS, en buena medida, porque ya se han realizado excelentes estudios al respecto como el de Diego Gracia (2007), Llenín (1990), Sáez Cruz (1995), Cabría (1997), Solari (2010), etc. Lo que aquí se pretende es actualizar la marcha de la "prueba» de Dios de Zubiri (la vía teísta), para lo cual sería importante analizar cómo se entiende en IS el razonamiento y la razón. Veamos esto en pasos sucesivos.

a) Ante todo, hay que explicar qué se entiende por razón. Zubiri pretende en IS hacer un fino análisis de la aprehensión humana, donde muestra tres modos de una misma intelección sentiente, cada uno fundado en el anterior: la aprehensión primordial, el logos y la razón. Para nuestro tema nos interesa destacar más el papel de la razón. La razón nos «actualiza» o hace presente lo real desde su fundamento. ¿Qué es fundamento? Zubiri nos dice: «fundamento es todo aquello que determina desde sí mismo, pero en y por sí mismo, a lo percibido, de suerte que éste es una realización del fundamento en lo fundado mismo ${ }^{28}$. Se busca, entonces, «desde lo percibido mismo», aquello que determina por qué es como es la cosa real percibida. Esta búsqueda de fundamento es, para Zubiri, algo que nos es dado por la aprehensión misma de realidad. Lo que «no» nos es dado es «cuál» sea el «contenido concreto» del fundamento de lo real. Esta búsqueda es, justamente, el conocimiento. El conocimiento es búsqueda de contenido fundamental. Este conocimiento posee una estructura con tres momentos. En primer lugar, hay que atender a «lo que» se conoce. Decíamos que la razón busca el «contenido del fundamento» de lo percibido. Ese momento fundante de la realidad es lo que Zubiri llama «realidad-fundamento». No es una realidad que «además» fundamente, sino que su modo de presentación consiste en fundar. Pero no hay fundamento sin algo fundado. Esa realidad fundada es lo que Zubiri llama objeto o realidadobjeto. Realidad-objeto y realidad-fundamento son los dos modos en que se nos actualiza lo real en la razón. En segundo lugar, el conocimiento posee intrínsecamente un «método». Para Zubiri, método «es un abrirse paso hacia el fundamento. Método es por tanto vía del conocer en cuanto tal» ${ }^{29}$. El método como «vía» racional posee, a su vez, tres momentos: un «sistema de referencia» (el punto de partida), un «esbozo» de lo que posiblemente «podría»

28 ZuBIRI, X. (1983). Inteligencia y razón. Madrid: Alianza, p. 161.

29 Ibíd., p. 203. 
ser el contenido de tal fundamento y la «experiencia» como probación física o tanteo de ese esbozo. Por último, en tercer lugar, el conocimiento posee un momento de «verificación». Hay que ver si se va cumpliendo o no lo esbozado en la experiencia.

b) En segundo lugar, debemos preguntarnos ¿qué es un razonamiento? Básicamente, en un razonamiento se trata de inferir lógicamente un juicio (conclusión) a partir de otros juicios (premisas). Se suele decir que hay dos grandes tipos de razonamiento; el deductivo y el inductivo. Zubiri mismo niega que exista, en rigor, el razonamiento inductivo ${ }^{30}$. Ahora bien, la prueba de la existencia de Dios de Zubiri, según lo expresado hasta antes del curso de 1973, parece ser un tipo de razonamiento deductivo. De este tipo parecen ser también otras pruebas de Dios en la historia de la filosofía como, por ejemplo, las vías de santo Tomás, por las cuales se afirma que la existencia de Dios «es demostrable por los efectos con que nos encontramos» ${ }^{31}$. A partir de los efectos (algo observable) demostramos, por ejemplo, su causa última. Este procedimiento demostrativo no debe ser minimizado. En las ciencias se suele usar también este procedimiento. Así, por ejemplo, se ha podido afirmar la existencia de un planeta desconocido (después llamado Neptuno) a partir de algunas anomalías observadas por el telescopio en la órbita de Urano. Dadas las observaciones de las posiciones de Urano y las leyes de la física de Newton (premisas), se infiere la existencia de un nuevo planeta que luego es descubierto por el telescopio. Aquí podemos ver cómo a partir de ciertas premisas que consideramos verdaderas podemos inferir otros juicios que "parecen» llevarnos a nuevos conocimientos. Sin embargo, como hemos visto, Zubiri comenzó poco a poco a distanciarse de entender su prueba como un mero razonamiento. Ante todo, como vimos, porque la prueba requiere también del momento de apropiación volitiva. Pero, en segundo lugar, y más radicalmente, porque la prueba no parece ser, en su aspecto intelectivo, claramente demostrativa. Es lo que se esboza, como hemos visto, en el curso de 1973. Pero Zubiri no aclara allí este punto. Por ello, se le hizo necesario, tal vez, repensar el razonamiento y su relación con la razón. Es lo que realizó en IS.

c) Para Zubiri, razón no es formalmente razonamiento o raciocinio. Así nos lo dice expresamente: "razón no es formalmente raciocinio, no es formalmente lo raciocinante. La razón razonante es tan sólo un tipo -y no el más importante- de razón ${ }^{32}$. Esto es muy importante de destacar porque, recordemos, la prueba o argumento de Dios se entendía antes del curso de 1973 como un proceso demostrativo o de razonamiento, proceso que Zubiri, recordemos, había calificado como el modo «más estricto y constringente de intelección $»^{33}$. Ahora se ha distinguido entre razón y razonamiento, dejando a este último proceso intelectual como algo distinto de la razón, aunque ya

\footnotetext{
30 Cf. Ibíd., p. 208.

31 Santo Tomás, (2007). Suma de Teología, op. cit., I q 2, a 2.

32 Zubiri, X. (1983). Inteligencia y razón, op. cit., p. 99.

33 ZubIRI, X. (2012). El hombre y Dios, op. cit., p. 282.
} 
precisaremos de qué manera. ¿Qué ha sucedido? Como hemos visto, la razón actualiza lo real desde su fundamento. La razón nos dice en qué consiste el contenido profundo de lo real, por ejemplo, en qué consiste el verde, etc. El razonamiento, en cambio, conecta unos juicios con otros, pero este proceso intelectual no actualiza por sí solo el contenido de lo real en profundidad. El razonamiento es, para Zubiri, una mera concatenación formal de juicios sin conexión con los «contenidos» de realidad. No nos actualiza la "verdad» racional $^{34}$. Por ello, todo razonamiento, en última instancia, puede reemplazarse por símbolos. El razonamiento tampoco es «vía» o método para actualizar lo real en su fundamento. Por ejemplo, la deducción le parece a Zubiri un razonamiento lógico legítimo, pero no es un método por sí solo, pues, por el mero hecho de razonar no actualizamos lo real desde su fundamento. Así, por ejemplo, la conclusión de un silogismo no es fuente por sí sola de nueva verdad racional. Volveré pronto sobre esto, para el caso del argumento de la existencia de Dios. La inducción, por su parte, como hemos visto, no le parece a Zubiri un razonamiento lógico legítimo, pero sí puede servir su conclusión como «esbozo» del método o vía racional. Zubiri nos lo explica así:

«Nunca se ha conseguido estructurar un razonamiento inductivo. [...] En cambio, la inducción existe como método estricto y riguroso. Se parte de lo real actualizado en hechos y se va por repetición (conforme a la ley de los grandes números) desde los resultados experimentales a un enunciado general. Este enunciado enuncia la actualización del fundamento» ${ }^{35}$.

Así pues, yo puedo obtener un enunciado universal por inducción y usarlo como esbozo de conocimiento. Así, por ejemplo, por la observación de varios cisnes blancos podría inferirse inductivamente que «todos los cisnes son blancos». A partir de ello, puedo afirmar como esbozo que el fundamento de los cisnes («ser blanco») es algo que constituye o funda a todo cisne. Sólo por este último momento nos encontramos, en rigor, ante una afirmación racional. Desde luego, tal afirmación es sólo un esbozo que debe «verificarse» en la experiencia. Dejemos de lado la veracidad de tal afirmación. Lo importante es destacar que un juicio es racional si actualiza lo real desde su fundamento, y no por ser obtenido como mero «resultado» de un razonamiento lógico, ya sea deductivo o inductivo.

Razón no es, pues, razonamiento. Esto no significa, por cierto, que Zubiri niegue que exista el razonamiento o que no tenga ningún papel en la actualización racional. Zubiri nos lo dice expresamente: «Esto no significa que en la intelección racional no haya razonamientos. Los hay y tiene que haberlos forzosamente, de la misma manera que hay juicios. Pretender lo contrario sería más que una imposibilidad una estupidez» ${ }^{36}$.

34 Cf. ZuBIRI, X. (1983). Inteligencia y razón, op. cit., p. 209.

35 Ibíd., pp. 208-209.

36 Ibíd., p. 209. 
d) Finalmente, hay que intentar pensar estas distinciones de razón y razonamiento, expresadas en IS, en la prueba o argumento de Dios. Ante todo, hay que señalar que la prueba de Dios es claramente un momento «racional» de la intelección sentiente, no es un puro momento de la aprehensión primordial ni del logos. El argumento de la existencia de Dios, por ejemplo, no surge por una mera descripción de lo aprehendido. No es «punto de partida» como el análisis de la religación. Además, hay que tener en cuenta que la prueba busca demostrar la «existencia» de Dios. Para Zubiri, la existencia es un momento del «contenido» de lo real más que de su «formalidad». Nos lo dice expresamente:

«Existencia y notas conciernen al contenido de lo real. Ciertamente la existencia no es una nota más del contenido. Pero no es ésta la cuestión. Porque aunque no sea una nota, la existencia es un momento que concierne formalmente al contenido de lo aprehendido, pero no es formalmente un momento de su realidad ${ }^{37}$.

Como la razón busca el «contenido» fundamental de lo real, lo que busca, ante todo, es actualizar la «existencia» de la realidad-fundamento y sus «notas» o caracteres fundamentales. Así, por ejemplo, la astronomía buscaba verificar desde Urano y su anomalía la «existencia» de Neptuno y sus notas como tamaño, color, etc.

Ahora bien, en virtud de todo lo dicho anteriormente, hay que señalar que tal prueba de la existencia de Dios no puede ser producto de un mero razonamiento. $\mathrm{La}$ «vía» de la religación no es una vía formalmente demostrativa. Ello porque la conclusión de un razonamiento, por sí sola, no nos lleva «directamente» a conocer el «contenido» de esa realidad-fundamento, es decir, su existencia y notas. Así, por ejemplo, el mero razonamiento sobre la «anomalía» de las posiciones de Urano no nos lleva a verificar directamente la «existencia» de un planeta X (Neptuno). Sólo nos lleva a su «posibilidad». Tal razonamiento descansa, ante todo, como supuesto, en la veracidad de sus premisas. Siempre es posible pensar en otra conclusión, si se cambian las premisas. La "anomalía» de Urano no sería tal si se hubiese cuestionado las leyes de Newton o las observaciones telescópicas. Pero aunque las premisas fueran ciertas, se requiere adicionalmente la "verificación» (aquí está el tercer momento de la razón) de la conclusión del argumento. En este caso astronómico, tal verificación se dio con las observaciones telescópicas de Neptuno (dejo de lado el problema de si la observación telescópica es suficiente para verificar una hipótesis astronómica). Hasta antes de tal verificación, sólo teníamos la «posibilidad» de su existencia, pues siempre hay otras posibilidades de explicar el punto de partida. El razonamiento, por sí solo, entonces, «no» lleva directamente al conocimiento del contenido o existencia de una realidad-fundamento. Ésta «parece» ser la precisión que tendría que hacer Zubiri a su argumento. La prueba no conduce directamente al contenido o existencia de Dios mismo, sino a su posibilidad.

37 ZuBIRI, X. (1980). Inteligencia sentiente, primera parte: Inteligencia y realidad. Madrid: Alianza, p. 192. 
Pensado desde el lenguaje de IS, tal vez habría que decir que todo el argumento sobre la existencia y concreción de esta realidad absolutamente absoluta (Dios) es, al parecer, un «esbozo», una «posibilidad» que debe probar su valía, su verificación en la experiencia, tal como las anomalías de Urano, según la física de Newton, nos llevan al «esbozo» de un nuevo planeta que deberá verificarse en la experiencia. Zubiri no lo dice expresamente, pero es algo que "podría» actualizarse desde IS, especialmente, desde Inteligencia y razón. Es la tesis que sostiene, por ejemplo, Diego Gracia ${ }^{38}$, y que después han seguido otros autores.

La prueba de Dios, entonces, para que se entienda como "esbozo», debe tener en cuenta cuatro cosas. Primero, el esbozo debe ser tomado como una «posibilidad». No es todavía realidad verificada. Segundo, esta posibilidad debe pretender actualizar lo real desde su fundamento. No es «mera» posibilidad sino posibilidad de fundamento. Tercero, no es necesario que el esbozo sea el resultado de una demostración o razonamiento. El esbozo puede ser incluso producto de una «libre construcción». Por ello, la prueba no necesita ser una demostración lógica rigurosa. Ya veíamos que los resultados de la inducción, que «no» son una demostración lógica rigurosa para Zubiri, sí pueden servir de esbozo. Tal vez, esto es lo que Zubiri empezó a pensar a partir del curso de 1973 sobre su «prueba» a la que califica, recordemos, como una «intelección procesual» en sentido amplio. Y, cuarto, que el esbozo, para ser probado, requiere de la experiencia (individual, social e histórica) por la cual lo vamos verificando o no. Esto lo dice espléndidamente Diego Gracia:

«El esbozo es lógico y, en el caso del acceso a Dios, consiste en la formulación de un argumento sobre Dios. Se trata de un argumento, no de una prueba. Por eso Zubiri le confiere sólo la categoría de "esbozo". Si fuera prueba, sobraría todo lo demás. Pero no sucede así. El argumento por sí solo no tiene la capacidad de llevarnos a Dios. Necesita de algo más, el momento de "experiencia". Dios, de ser algo, es experiencia, la experiencia del fundamento en nosotros. Es experiencia, dice Zubiri, de conformación y compenetración. Aquí sí cabe hablar de "prueba". Pero prueba en el sentido físico, no en el lógico. No se trata de probar lógicamente sino de probar en el sentido en que decimos que probamos un manjar; es "probación física" ${ }^{39}$.

La experiencia de Dios es, pues, una probación física o verificación de su esbozo, tal como probamos un manjar, pero no como el que simplemente lo degusta, sino como el que lo degusta con un esbozo previo de lo que sería en la realidad. Ello porque sin esbozo no hay experiencia, no hay nada que probar. Tal esbozo sería, en nuestro caso, el argumento de Dios que Zubiri elabora desde la vía de la religación.

38 Cf. Gracia, D. (2007). Voluntad de verdad. Madrid: Triacastela, pp. 222-229.

39 Gracia, D. (2003). «Zubiri y la experiencia teologal» en La filosofía como Pasión, Homenaje a Jorge Eduardo Rivera Cruchaga en su 75 cumpleaños. Madrid: Trotta, p. 255. 


\section{CONCLUSIÓN}

Zubiri meditó muchas veces, en sus últimos años, sobre su «prueba» de Dios. Poco a poco la fue entendiendo como algo que no es obtenido por un mero razonamiento o proceso demostrativo. Primero, porque toda prueba de Dios lleva ineludiblemente un aspecto volitivo, tanto en el momento intelectivo como en el de entrega. Y segundo, y más radical para nuestro tema, porque un razonamiento, por sí solo, no nos lleva «directamente» al conocimiento concreto de Dios (existencia y notas). Se requiere de la experiencia, del probar o verificar el esbozo en la experiencia individual, social e histórica. La prueba demostrativa no verifica directamente el contenido profundo de Dios tal como las posiciones de Urano no verifican directamente el contenido o existencia de Neptuno. La razón busca el «contenido» de la realidad-fundamento. Este contenido último no se obtiene pasivamente desde la aprehensión primordial o logos, o en virtud de un mero razonamiento, sino que debe «esbozarse» o «crearse» como posibilidad e intentar verificarse en la experiencia.

Ahora bien, hay que precisar que, si bien la prueba no nos deja directamente, como punto de llegada, en el «contenido» de la realidad-fundamento, sí parte del momento de «realidad» de esta realidad-fundamento dada en aprehensión primordial y logos. La prueba o argumento de Dios intenta ir probando el "contenido» de la realidad-fundamento en que "ya» estamos. El argumento de la existencia de Dios en Zubiri, por tanto, no pretende basarse en lo que «sabemos» de Dios por revelación. No pretende verificar, por ejemplo, que Dios es creador del mundo. El argumento se basa en una experiencia de realidad-fundamento como Dios que intentamos probar a partir de nuestros esbozos. La prueba nace del esbozo creado desde la realidad aprehendida y vuelve a ella para su probación o verificación. La prueba, en cierto modo, viene y va hacia Dios. Viene de Dios como realidad-fundamento. Y va hacia Dios como realidad absolutamente absoluta. Esto es lo que tiene que "probar» la experiencia. Aunque Zubiri no completó su «prueba», creemos que en este artículo hay suficientes pistas obtenidas desde el estudio de sus últimos escritos que apuntan, como un vector, hacia lo expresado finalmente en IS, y que dan mayor fundamento a lo expresado por otros filósofos sobre la tesis de la prueba de Dios como esbozo en el último Zubiri.

\section{BiBLIOGRAFÍA}

CABRía, J. (1997). Relación Teología-Filosofía en el pensamiento de Xavier Zubiri. Roma: Gregorian University Press.

Gracia, D. (2007). Voluntad de verdad. Para leer a Zubiri. Madrid: Triacastela.

Gracia, D. (2003). "Zubiri y la experiencia teologal», en La filosofía como Pasión. Homenaje a Jorge Eduardo Rivera Cruchaga en su 75 cumpleaños. (Patricio Brickle, ed.), Madrid: Trotta, pp. 249-263. 
Llenín, F. (1990). La realidad divina. El problema de Dios en Xavier Zubiri, Oviedo: Seminario Metropolitano.

SÁEz Cruz, J. (1995). La accesibilidad de Dios: su mundanidad y transcendencia en X. Zubiri. Salamanca: Univ. Pontificia de Salamanca.

Santo Tomás. (2007). Suma de Teología. Madrid: BAC.

SOLARI, E. (2010). La raíz de lo sagrado. Contribuciones de Zubiri a la filosofía de la religión. Santiago: RIL.

VARgas, E. (2015). «Filosofía y el problema de Dios», en Pensamiento, vol. 71, núm. 266, pp. 491-504.

ZuBIRI, X. (1980). Inteligencia sentiente, primera parte: Inteligencia y realidad. Madrid: Alianza.

-. (1983). Inteligencia y razón. Madrid: Alianza.

-. (1987). Naturaleza, Historia, Dios. Madrid: Alianza.

-. (1984). El hombre y Dios. Primera Edición. Madrid: Alianza.

-. (2012). El hombre y Dios. Nueva edición. Madrid: Alianza.

-. (2015). El problema teologal del hombre: Dios, religión, cristianismo. Madrid: Alianza.

Pontificia Universidad Católica de Valparaíso, Chile esteban.vargas@pucv.cl

ESTEBAN VARGAS

[Artículo aprobado para publicación en noviembre de 2013] $]^{40}$

40 Nota editorial: Recordamos que este artículo, como todos los publicados en PensaMIENTO, fue revisado antes de su edición por el autor, realizando en este caso una pertinente actualización bibliográfica 
